\title{
Higher order numerical schemes for affinely controlled nonlinear systems*
}

\author{
L. Grüne and P. E. Kloeden \\ Fachbereich Mathematik, \\ Johann Wolfgang Goethe Universität, \\ D-60054 Frankfurt am Main, Germany \\ E-mail: gruene@math.uni-frankfurt.de, \\ kloeden@math.uni-frankfurt.de
}

February 10, 2000

\begin{abstract}
A systematic method for the derivation of high order schemes for affinely controlled nonlinear systems is developed. Using an adaptation of the stochastic Taylor expansion for control systems we construct Taylor schemes of arbitrary high order and indicate how derivative free Runge-Kutta type schemes can be obtained. Furthermore an approximation technique for the multiple control integrals appearing in the schemes is proposed.
\end{abstract}

\section{AMS Subject Classification: 65L05, 93B40}

Key words: Affine control systems, Taylor expansion, Taylor schemes, Runge-Kutta type schemes, multiple control integrals.

\section{Introduction}

Traditional numerical schemes for ordinary differential equations, such as Runge-Kutta schemes, usually fail to attain their asserted order when applied to ordinary differential control equations due to the measurability of the control functions. A similar situation occurs with stochastic differential equations due to the nondifferentiability of the

*This work was supported by the DFG Forschungschwerpunkt "Ergodentheorie, Analysis und effiziente Simulation dynamischer Systeme". 
driving noise processes. To construct higher order numerical schemes for stochastic differential equations, one needs to start with an appropriate stochastic Taylor expansion to ensure consistency with the less robust stochastic calculus as well as a higher order of convergence. This is the opposite procedure to that used for numerical schemes for ordinary differential equations, where heuristic arguments are typically used to derive a scheme and the Taylor expansion is then used to establish its local discretization order.

In this paper we will show that an analogous approach to that in the stochastic case enables one to derive one-step numerical schemes of an arbitrary desired order for affinely controlled nonlinear systems. In particular, we will first formulate, and then apply to construct numerical schemes, the general Taylor expansion of a function $F(t, x(t))$ with respect to the solutions of an $d$-dimensional affinely controlled nonlinear system with $m$-dimensional control functions of the form

$$
\frac{d x}{d t}=f^{0}(t, x)+\sum_{j=1}^{m} f^{j}(t, x) u^{j}(t),
$$

where $t \in\left[t_{0}, T\right]$ and $x=\left(x^{1}, \ldots, x^{m}\right) \in \mathbb{R}^{d}$, and the control functions $u(t)=\left(u^{1}(t), \ldots\right.$, $\left.u^{m}(t)\right)$ are measurable and take values in a compact convex subset $\mathbf{U}_{m}$ of $\mathbb{R}^{m}$. Our expansion is essentially the same as the Fliess expansion that is well known in control theory [9], with the main difference lying in the compact notation that we adapt from stochastic calculus [13], which allows, in particular, a transparent representation of the remainder term and a systematic and straightforward derivation of approximations of an arbitrary desired order. Some of these schemes had already been derived by Ferretti [5] for a restricted class of systems of the form (1), starting from a traditional Runge-Kutta scheme and then modifying it with the help of a Fliess expansion.

Numerical schemes for affinely controlled systems have recently received considerable interest, since complex nonlinear control systems do in general not allow an analytic solution and hence require numerical treatment for both analysis and controller design. See for instance the monograph [2] for a number of algorithms for this class of systems, where in each of them the approximation of trajectories appears as a subproblem.

The organization of this paper is as follows. We start with an illustrative example of our Taylor expansions in Section 2, which is followed by the introduction of the necessary notation in Section 3 and the precise statement of the general Taylor expansion in Section 4. In Section 5 we explain how Taylor approximations of arbitrary desired order can be obtained from this expansion, which we then use in Section 6 for the construction of numerical Taylor schemes of arbitrary order. In Section 7 we show how derivative-free schemes can be obtained from these Taylor schemes, thus providing a means for the construction of the right kind of "Runge-Kutta" schemes for the affinely 
controlled nonlinear systems (1). Several simplifications to the Taylor schemes based on a special additive or commutative control structure of the system (1) are also indicated in Section 8. The approximation of the multiple control integrals appearing in the schemes is then addressed in Section 9, in particular approximation by averaging for a single control function and then the approximation of the set of multiple control integrals for all measurable control functions. Finally, we illustrate our results by a numerical example in Section 10.

\section{An illustrative example}

We consider the solution $x(t)$ of the 1-dimensional affinely controlled autonomous differential equation

$$
\frac{d x}{d t}=f^{0}(x)+f^{1}(x) u(t)
$$

which is interpreted in the sense of Carathéodory, or its equivalent integral equation representation

$$
x(t)=x\left(t_{0}\right)+\int_{t_{0}}^{t} f^{0}(x(s)) d s+\int_{t_{0}}^{t} f^{1}(x(s)) u(s) d s
$$

for $t \in\left[t_{0}, T\right]$, where the coefficients $f^{0}$ and $f^{1}$ in (2) are sufficiently smooth real-valued functions satisfying a linear growth bound and the control function $u(t)$ is measurable and takes values in a compact interval $\mathbf{U}_{1}=\left[u_{\min }, u_{\max }\right]$.

Then, for any continuously differentiable function $F: \mathbb{R} \rightarrow \mathbb{R}$ the chain rule for the absolutely continuous solutions of equations (1) [6] gives

$$
\begin{aligned}
F(x(t))=F\left(x\left(t_{0}\right)\right) & +\int_{t_{0}}^{t}\left(f^{0}(x(s)) \frac{\partial}{\partial x} F(x(s))\right) d s \\
& +\int_{t_{0}}^{t} f^{1}(x(s)) \frac{\partial}{\partial x} F(x(s)) u(s) d s \\
=F\left(x\left(t_{0}\right)\right) & +\int_{t_{0}}^{t} L^{0} F(x(s)) d s+\int_{t_{0}}^{t} L^{1} F(x(s)) u(s) d s,
\end{aligned}
$$

for $t \in\left[t_{0}, T\right]$, where the operators $L^{0}$ and $L^{1}$ are defined by

$$
L^{0}=f^{0} \frac{\partial}{\partial x}, \quad L^{1}=f^{1} \frac{\partial}{\partial x} .
$$

Obviously, for $F(x) \equiv x$ we have $L^{0} F=f^{0}$ and $L^{1} F=f^{1}$, in which case (3) reduces to the original affinely controlled differential equation (2), that is to

$$
x(t)=x\left(t_{0}\right)+\int_{t_{0}}^{t} f^{0}(x(s)) d s+\int_{t_{0}}^{t} f^{1}(x(s)) u(s) d s .
$$


If we now apply the chain rule (3) to each of the functions $F=f^{0}$ and $F=f^{1}$ in (2) we obtain

$$
\begin{aligned}
x(t)= & x\left(t_{0}\right)+\int_{t_{0}}^{t}\left(f^{0}\left(x\left(t_{0}\right)\right)+\int_{t_{0}}^{s} L^{0} f^{0}(x(r)) d r+\int_{t_{0}}^{s} L^{1} f^{0}(x(z)) u(z) d z\right) d s \\
& +\int_{t_{0}}^{t}\left(f^{1}\left(x\left(t_{0}\right)\right)+\int_{t_{0}}^{s} L^{0} f^{1}(x(z)) d z+\int_{t_{0}}^{s} L^{1} f^{1}(x(z)) u(z) d z\right) u(s) d s \\
= & x\left(t_{0}\right)+f^{0}\left(x\left(t_{0}\right)\right) \int_{t_{0}}^{t} d s+f^{1}\left(x\left(t_{0}\right)\right) \int_{t_{0}}^{t} u(s) d s+R
\end{aligned}
$$

with the remainder

$$
\begin{aligned}
R= & \int_{t_{0}}^{t} \int_{t_{0}}^{s} L^{0} f^{0}(x(z)) d z d s+\int_{t_{0}}^{t} \int_{t_{0}}^{s} L^{1} f^{0}(x(z)) u(z) d z d s \\
& +\int_{t_{0}}^{t} \int_{t_{0}}^{s} L^{0} f^{1}(x(z)) u(s) d z d s+\int_{t_{0}}^{t} \int_{t_{0}}^{s} L^{1} f^{1}(x(z)) u(z) u(s) d z d s .
\end{aligned}
$$

This is the simplest nontrivial Taylor expansion for the affinely controlled system (2).

We can continue the procedure, for instance, by applying the chain rule (3) to $F=$ $L^{1} f^{1}$ in (4), in which case we get

$$
\begin{gathered}
x(t)=x\left(t_{0}\right)+f^{0}\left(x\left(t_{0}\right)\right) \int_{t_{0}}^{t} d s+f^{1}\left(x\left(t_{0}\right)\right) \int_{t_{0}}^{t} u(s) d s \\
+L^{1} f^{1}\left(x\left(t_{0}\right)\right) \int_{t_{0}}^{t} \int_{t_{0}}^{s} u(z) u(s) d z d s+\bar{R}
\end{gathered}
$$

with remainder

$$
\begin{aligned}
\bar{R}= & \int_{t_{0}}^{t} \int_{t_{0}}^{s} L^{0} f^{0}(x(z)) d z d s+\int_{t_{0}}^{t} \int_{t_{0}}^{s} L^{1} f^{0}(x(z)) u(z) d z d s \\
& +\int_{t_{0}}^{t} \int_{t_{0}}^{s} L^{0} f^{1}(x(z)) u(s) d z d s+\int_{t_{0}}^{t} \int_{t_{0}}^{s} \int_{t_{0}}^{z} L^{0} L^{1} f^{1}(x(r)) d r u(z) u(s) d z d s \\
& \quad+\int_{t_{0}}^{t} \int_{t_{0}}^{s} \int_{t_{0}}^{z} L^{1} L^{1} f^{1}(x(r)) u(r) u(z) u(s) d r d z d s .
\end{aligned}
$$

Later we shall formulate the Taylor expansions (there are many possibilities) for a general function $F$ and arbitrarily high order. Nevertheless, its main properties are already apparent in the preceding example. In particular, we have an expansion with the multiple control integrals

$$
\int_{t_{0}}^{t} d s, \quad \int_{t_{0}}^{t} u(s) d s, \quad \int_{t_{0}}^{t} \int_{t_{0}}^{s} u(z) d z u(s) d s
$$

and a remainder term involving the next multiple control integrals, but now with nonconstant integrands. The Taylor expansions obtained in this way thus generalize, 
and include as a special case, the usual Taylor formula, i.e. take $f^{0} \equiv 1$ and the other $f^{j} \equiv 0$. They are essentially the same as truncated versions of the infinite Fliess expansions that are well known in control theory [9], however, the notation adapted from stochastic Taylor expansions [13] allows arbitrarily order expansions to be written out very compactly and transparently, in particular yielding an explicit expression for the remainder term and allowing straightforward derivation of arbitrary order approximations. Moroever, they do not require any restrictions on the form of the $f^{0}$ and $f^{1}$ coefficients such as a constant $f^{1}$ in [5] apart from the necessary smoothness up to a certain order $N \in \mathbb{N}$.

\section{Multi-indices and multiple integrals}

In the following sections we shall refer to the nonautonomous $d$-dimensional affinely controlled differential equation (1), which we rewrite in the equivalent integral form

$$
x(t)=x\left(t_{0}\right)+\int_{t_{0}}^{t} f^{0}(s, x(s)) d s+\sum_{j=1}^{m} \int_{t_{0}}^{t} f^{j}(s, x(s)) u^{j}(s) d s
$$

or even more compactly as

$$
x(t)=x\left(t_{0}\right)+\sum_{j=0}^{m} \int_{t_{0}}^{t} f^{j}(s, x(s)) u^{j}(s) d s
$$

where we have introduced a fictitious control function $u^{0}(t) \equiv 1$ so that the first integral term can be included in the summation, which will be notationally very convenient in what follows.

\subsection{Multi-indices}

Let $m \geq 0$ correspond to the number of components of the control functions under consideration. We call a row vector

$$
\alpha=\left(j_{1}, j_{2}, \ldots, j_{l}\right)
$$

where $j_{i} \in\{0,1, \ldots, m\}$ for $i=1, \ldots, l$, a multi-index of length $l:=l(\alpha) \geq 1$ and for completeness we write $\odot$ for the multi-index of length zero, that is, with $l(\odot)=0$. We denote the set of all such multi-indices by $\mathcal{M}_{m}$, so

$$
\mathcal{M}_{m}=\left\{\left(j_{1}, j_{2}, \ldots, j_{l}\right): j_{i} \in\{0,1, \ldots, m\}, i \in\{1, \ldots, l\} \quad \text { for } l=1,2,3, \ldots\right\} \cup\{\odot\} .
$$


For any $\alpha=\left(j_{1}, j_{2}, \ldots, j_{l}\right) \in \mathcal{M}_{m}$ with $l(\alpha) \geq 1$, denote by $-\alpha$ and $\alpha-$ for the multi-index in $\mathcal{M}_{m}$ obtained by deleting the first and the last component, respectively, of $\alpha$, thus

$$
-\alpha=\left(j_{2}, \ldots, j_{l}\right) \quad \alpha-=\left(j_{1}, \ldots, j_{l-1}\right) .
$$

In addition, define the concatenation of any two multi-indices $\alpha=\left(j_{1}, j_{2}, \ldots, j_{k}\right)$ and $\bar{\alpha}=\left(\bar{j}_{1}, \bar{j}_{2}, \ldots, \bar{j}_{l}\right)$ in $\mathcal{M}_{m}$ by

$$
\alpha * \bar{\alpha}=\left(j_{1}, j_{2}, \ldots, j_{k}, \bar{j}_{1}, \bar{j}_{2}, \ldots, \bar{j}_{l}\right),
$$

that is, the multi-index formed by adjoining the two given multi-indices. Finally, define $n(\alpha)$ to be the number of components of a multi-index $\alpha \in \mathcal{M}_{m}$ that are equal to 0 .

\subsection{Multiple Control Integrals}

For a multi-index $\alpha=\left(j_{1}, j_{2}, \ldots, j_{l}\right) \in \mathcal{M}_{m}$, some integrable control function $u$ : $\mathbb{R} \rightarrow \mathbf{U}_{m}$ and an integrable function $f:\left[t_{0}, T\right] \rightarrow \mathbb{R}$ we define the multiple integral $I_{\alpha}[f(\cdot)]_{t_{0}, t}$ recursively by

$$
I_{\alpha}[f(\cdot)]_{t_{0}, t}:=\left\{\begin{array}{cc}
f(t) & : \quad l=0 \\
\int_{t_{0}}^{t} I_{\alpha-}[f(\cdot)]_{t_{0}, s} u^{j_{l}}(s) d s & : \quad l \geq 1
\end{array} .\right.
$$

We note that $I_{\alpha}[f(\cdot)]_{t_{0}, \cdot}:\left[t_{0}, T\right] \rightarrow \mathbb{R}$ is continuous, hence integrable, so the integrals are well defined. Hence, for example

$$
\begin{aligned}
I_{\odot}[f(\cdot)]_{t_{0}, t} & =f(t), \quad I_{(0)}[f(\cdot)]_{t_{0}, t}=\int_{t_{0}}^{t} f(s) d s, \quad I_{(1)}[f(\cdot)]_{t_{0}, t}=\int_{t_{0}}^{t} f(s) u^{1}(s) d s, \\
I_{(0,1)}[f(\cdot)]_{0, t} & =\int_{0}^{t} \int_{0}^{s_{2}} f\left(s_{1}\right) u^{1}\left(s_{2}\right) d s_{1} d s_{2} \\
I_{(0,2,1)}[f(\cdot)]_{0, t} & =\int_{0}^{t} \int_{0}^{s_{3}} \int_{0}^{s_{2}} f\left(s_{1}\right) u^{2}\left(s_{2}\right) u^{1}\left(s_{3}\right) d s_{1} d s_{2} d s_{3} .
\end{aligned}
$$

For simpler notation, we shall often abbreviate $I_{\alpha}[f(\cdot)]_{t_{0}, t}$ to $I_{\alpha, t}$ or just $I_{\alpha}$ when $f(t)$ $\equiv 1$ and shall explicitly write $I_{\alpha, u}[f(\cdot)]_{t_{0}, t}, I_{\alpha, u, t}$ or $I_{\alpha, u}$ when we want to emphasize a specific control function $u$ 


\subsection{Coefficient Functions}

For each $\alpha=\left(j_{1}, \ldots, j_{l}\right) \in \mathcal{M}_{m}$ and function $F:\left[t_{0}, T\right] \times \mathbb{R}^{d} \rightarrow \mathbb{R}$, the coefficient function $F_{\alpha}$ is define recursively by

$$
F_{\alpha}=\left\{\begin{array}{ll}
F & : \quad l=0 \\
L^{j_{1}} F_{-\alpha} & : \quad l \geq 1
\end{array},\right.
$$

where the partial differential operators are defined by

$$
L^{0}=\frac{\partial}{\partial t}+\sum_{k=1}^{d} f^{0, k} \frac{\partial}{\partial x^{k}}, \quad L^{j}=\sum_{k=1}^{d} f^{j, k} \frac{\partial}{\partial x^{k}}, \quad j=1, \ldots, m .
$$

This definition requires the functions $F, f^{0}, f^{1}, \ldots, f^{m}$ to be sufficiently smooth.

For example, in the autonomous scalar dimensional case with $d=m=1$ for the identity function $F(t, x) \equiv x$ we have

$$
\begin{gathered}
F_{(0)}=f^{0}, \quad F_{\left(j_{1}\right)}=f^{j_{1}}, \quad F_{(0,0)}=f^{0} f^{0 \prime} \\
F_{\left(0, j_{1}\right)}=f^{0} f^{j_{1} \prime}, \quad F_{\left(j_{1}, 0\right)}=f^{0 \prime} f^{j_{1}}, \quad F_{\left(j_{1}, j_{2}\right)}=f^{j_{1}} f^{j_{2} \prime},
\end{gathered}
$$

where the dash ' denotes differentiation with respect to $x$.

When the function $F$ is not explicitly stated in the text we shall always take it to be the identity function $F(t, x) \equiv x$.

\subsection{Hierarchical and Remainder Sets}

Since different integrals can be expanded in forming a Taylor expansion, the terms with constant integrands cannot be written down completely arbitrarily. Rather, the set of corresponding multi-indices must form an hierarchical set.

A subset $\mathcal{A} \subset \mathcal{M}_{m}$ is called an hierarchical set if $\mathcal{A}$ is nonempty, if the multi-indices in $\mathcal{A}$ are uniformly bounded in length, that is $\sup _{\alpha \in \mathcal{A}} l(\alpha)<\infty$, and if

$$
-\alpha \in \mathcal{A} \text { for each } \alpha \in \mathcal{A} \backslash\{\odot\}
$$

where $\odot$ is the multi-index of length zero.

Thus, if a multi-index $\alpha$ belongs to an hierarchical set, then so does the multi-index $-\alpha$ obtained by deleting the first component of $\alpha$.

The remainder term of a Taylor expansion constructed with a given hierarchical set $\mathcal{A}$ involves only those multiple control integrals with multi-indices belonging to the corresponding remainder set $\mathcal{B}(\mathcal{A})$ which is defined by

$$
\mathcal{B}(\mathcal{A})=\left\{\alpha \in \mathcal{M}_{m} \backslash \mathcal{A}:-\alpha \in \mathcal{A}\right\}
$$


It thus consists of all of the next following multi-indices with respect to the given hierarchical set that do not already belong to the hierarchical set and is formed simply by adding a further component taking all possible values at the beginning of the "maximal" multi-indices in the hierarchical set.

\section{Taylor expansions for affine control systems}

We now formulate the Taylor expansion for the $d$-dimensional affinely controlled system (6) using the terminology that was introduced in the preceding section.

Theorem 1 Let $F: \mathbb{R}^{+} \times \mathbb{R}^{d} \rightarrow \mathbb{R}$ and let $\mathcal{A} \subset \mathcal{M}_{m}$ be an hierarchical set with remainder set $\mathcal{B}(\mathcal{A})$. Then the following Taylor expansion corresponding to the hierarchical set $\mathcal{A}$

$$
F(t, x(t))=\sum_{\alpha \in \mathcal{A}} I_{\alpha}\left[F_{\alpha}\left(t_{0}, x\left(t_{0}\right)\right)\right]_{t_{0}, t}+\sum_{\alpha \in \mathcal{B}(\mathcal{A})} I_{\alpha}\left[F_{\alpha}(\cdot, x(\cdot)),\right]_{t_{0}, t}
$$

holds, provided all of the derivatives of $F, f^{0}, f^{1}, \ldots, f^{m}$ and all of the multiple control integrals appearing here exist.

Proof: We give a sketch of the proof following that of the Ito-Taylor expansion [13, Theorem 5.5.1].

First we apply the integrated version of the chain rule for the types of functions under consideration [6], that is

$$
F(t, x(t))=F\left(t_{0}, x\left(t_{0}\right)\right)+\sum_{j=0}^{m} I_{(j)}\left[L^{(j)} F(\cdot, x(\cdot))\right]_{t_{0}, t},
$$

to the function $F_{\alpha}$ for some multi-index $\alpha \in \mathcal{A}$ to obtain

$$
\begin{aligned}
I_{\alpha}\left[F_{\alpha}(\cdot, x(\cdot))\right]_{t_{0}, t} & =I_{\alpha}\left[F_{\alpha}\left(t_{0}, x\left(t_{0}\right)\right)\right]_{t_{0}, t}+I_{\alpha}\left[\sum_{j=0}^{m} I_{(j)}\left[L^{(j)} F_{\alpha}(\cdot, x(\cdot))\right]_{t_{0}, \cdot}\right]_{t_{0}, t} \\
& =F_{\alpha}\left(t_{0}, x\left(t_{0}\right)\right) I_{\alpha, t_{0}, t}+\sum_{j=0}^{m} I_{(j) * \alpha}\left[F_{(j) * \alpha}(\cdot, x(\cdot))\right]_{t_{0}, t}
\end{aligned}
$$

We shall verify the expression in the theorem by induction over $k:=\max \{l(\alpha) \mid \alpha \in \mathcal{A}\}$. For $k=0$, the hierarchical set is simply $\mathcal{A}=\{\odot\}$, so the assertion follows directly from (13). For $k \geq 1$ consider the hierarchical set $\mathcal{E}:=\{\alpha \in \mathcal{A} \mid l(\alpha) \leq k-1\}$. Then

$$
F(t, x(t))=\sum_{\alpha \in \mathcal{E}} I_{\alpha}\left[F\left(t_{0}, x\left(t_{0}\right)\right)\right]_{t_{0}, t}+\sum_{\alpha \in \mathcal{B}(\mathcal{E})} I_{\alpha}[F(\cdot, x(\cdot))]_{t_{0}, t}
$$


holds by the induction assumption and, since by the definition of a remainder set we know that $\mathcal{A} \backslash \mathcal{E} \subseteq \mathcal{B}(\mathcal{E})$, we can conclude

$$
\begin{aligned}
F(t, x(t))= & \sum_{\alpha \in \mathcal{E}} I_{\alpha}\left[F\left(t_{0}, x\left(t_{0}\right)\right)\right]_{t_{0}, t} \\
& \quad+\sum_{\alpha \in \mathcal{A} \backslash \mathcal{E}} I_{\alpha}[F(\cdot, x(\cdot))]_{t_{0}, t}+\sum_{\alpha \in \mathcal{B}(\mathcal{A}) \backslash(\mathcal{A} \backslash \mathcal{E})} I_{\alpha}[F(\cdot, x(\cdot))]_{t_{0}, t} \\
= & \sum_{\alpha \in \mathcal{E}} I_{\alpha}\left[F\left(t_{0}, x\left(t_{0}\right)\right)\right]_{t_{0}, t}+\sum_{\alpha \in \mathcal{B}_{1}} I_{\alpha}[F(\cdot, x(\cdot))]_{t_{0}, t}
\end{aligned}
$$

with the last equality following from (14). Finally, since the definition of a remainder set implies that $\mathcal{B}_{1}=\mathcal{B}(\mathcal{A})$, we obtain the desired expression.

For example, in the general case with the hierarchical and remainder sets

$$
\mathcal{A}=\{\odot\}, \quad \mathcal{B}(\{\odot\})=\{(0), \cdots,(m)\},
$$

the Taylor expansion is

$$
\begin{aligned}
F(t, x(t)) & =I_{\odot}\left[F_{\odot}\left(t_{0}, x\left(t_{0}\right)\right)\right]_{t_{0}, t}+\sum_{\alpha \in \mathcal{B}(\{v\})} I_{\alpha}\left[F_{\alpha}(\cdot, x(\cdot))\right]_{t_{0}, t} \\
& =F\left(t_{0}, x\left(t_{0}\right)\right)+\int_{t_{0}}^{t} L^{0} F(s, x(s)) d s+\sum_{j=1}^{m} \int_{t_{0}}^{t} L^{j} F(s, x(s)) u^{j}(s) d s
\end{aligned}
$$

As another example, in the autonomous scalar case $d=m=1$ with $F(t, x) \equiv x$ and the hierarchical and remainder sets

$$
\mathcal{A}=\left\{\alpha \in \mathcal{M}_{1}: l(\alpha) \leq 2\right\}, \quad \mathcal{B}(\mathcal{A})=\left\{\alpha \in \mathcal{M}_{1}: l(\alpha)=3\right\}
$$

the Taylor expansion reads

$$
\begin{gathered}
x(t)=x\left(t_{0}\right)+f^{0} I_{(0)}+f^{1} I_{(1)}+f^{0} f^{0 \prime} I_{(0,0)}+f^{0} f^{1 \prime} I_{(0,1)} \\
+f^{1} f^{0 \prime} I_{(1,0)}+f^{1} f^{1 \prime} I_{(1,1)}+R_{3}\left(t, t_{0}\right)
\end{gathered}
$$

where the integrals are over the interval $\left[t_{0}, t\right]$, the coefficient functions here are all evaluated at $\left(t_{0}, x_{0}\right)$, the dash ' denotes differentiation with respect to $x$, and $R_{3}\left(t, t_{0}\right)$ is the corresponding remainder term.

\section{Taylor Approximations}

Taylor approximations of arbitrary higher order can be constructed by including in an appropriate way more terms from the Taylor expansions that are then truncated. We 
show here that a Taylor approximation of order $N=1,2, \ldots$ needs all of the multiple control integral terms from the Taylor expansion of up to and including order $N$, i.e. with the constant coefficients $F_{\alpha}\left(t_{0}, x\left(t_{0}\right)\right)$ and the corresponding multiple control integrals

$$
I_{\alpha, t_{0}, t_{0}+\Delta}=\int_{t_{0}}^{t_{0}+\Delta} \int_{t_{0}}^{s_{l}} \ldots \int_{t_{0}}^{s_{2}} u^{j_{1}}\left(s_{1}\right) \ldots u^{j_{l-1}}\left(s_{l-1}\right) u^{j_{l}}\left(s_{l}\right) d s_{1} \ldots d s_{l}
$$

for all multi-indices $\alpha$ in the hierarchical set

$$
\Gamma_{N}=\left\{\alpha \in \mathcal{M}_{m}: l(\alpha) \leq N\right\}
$$

Thus in the general multi-dimensional case $d, m=1,2, \ldots$ the Taylor approximation for $N=1,2,3, \ldots$ is defined by

$$
\begin{aligned}
F_{N}\left(t_{0}, x\left(t_{0}\right), \Delta\right) & :=\sum_{\alpha \in \Gamma_{N}} F_{\alpha}\left(t_{0}, x\left(t_{0}\right)\right) I_{\alpha, t_{0}, t_{0}+\Delta} \\
& =F\left(t_{0}, x\left(t_{0}\right)\right)+\sum_{\alpha \in \Gamma_{N} \backslash\{\odot\}} F_{\alpha}\left(t_{0}, x\left(t_{0}\right)\right) I_{\alpha, t_{0}, t_{0}+\Delta}
\end{aligned}
$$

with the coefficient functions $F_{\alpha}$ corresponding to the function $F(t, x)$.

Note that when the function $F(t, x)$ is $N+1$ times continuously differentiable and the drift and control coefficients $f^{0}, f^{1}, \ldots, f^{m}$ of the affinely controlled differential equation (6) are $N$ times continuously differentiable, then each of the integrals $I_{\alpha, t_{0}, t_{0}+\Delta}\left(F_{\alpha}(\cdot, x(\cdot))\right)$, that is

$$
\int_{t_{0}}^{t_{0}+\Delta} \int_{t_{0}}^{s_{l}} \ldots \int_{t_{0}}^{s_{2}} F_{\alpha}\left(s_{1}, x\left(s_{1}\right)\right) u^{j_{1}}\left(s_{1}\right) \ldots u^{j_{l-1}}\left(s_{l-1}\right) u^{j_{l}}\left(s_{l}\right) d s_{1} \ldots d s_{l}
$$

for $\alpha$ in the remainder set $\mathcal{B}\left(\Gamma_{N}\right)$ is of order $\Delta^{N+1}$. Since there are only finitely many, specifically $(m+1)$ !, remainder integrals, the truncation error here is

$$
\left|F_{N}\left(t_{0}, x\left(t_{0}\right), \Delta\right)-F\left(t_{0}+\Delta, x\left(t_{0}+\Delta\right)\right)\right| \leq K \Delta^{N+1},
$$

where the constant $K$ depends on $N$ as well as on a compact set containing the initial value $\left(t_{0}, x\left(t_{0}\right)\right)$ and the solution of the affinely controlled differential equation.

For the function $F(t, x) \equiv x^{k}$, the $k$ th component of the vector $x$, and $N=1,2$ and 3 , respectively, the solution $x\left(t_{0}+\Delta\right)$ of the controlled differential equation (6) satisfies the componentwise approximations

$$
\begin{aligned}
& x^{k}\left(t_{0}+\Delta\right)=x^{k}\left(t_{0}\right)+\sum_{j=0}^{m} f^{j, k}\left(t_{0}, x\left(t_{0}\right)\right) I_{(j)}+O\left(\Delta^{2}\right) \\
& x^{k}\left(t_{0}+\Delta\right)=x^{k}\left(t_{0}\right)+\sum_{j=0}^{m} f^{j, k}\left(t_{0}, x\left(t_{0}\right)\right) I_{(j)}+\sum_{j_{1}, j_{2}=0}^{m} L^{j_{1}} f^{j_{2}, j} I_{\left(j_{1}, j_{2}\right)}+O\left(\Delta^{3}\right)
\end{aligned}
$$


and

$$
\begin{aligned}
x^{k}\left(t_{0}+\Delta\right)=x^{k}\left(t_{0}\right) & +\sum_{j=0}^{m} f^{j, k}\left(t_{0}, x\left(t_{0}\right)\right) I_{(j)}+\sum_{j_{1}, j_{2}=0}^{m} L^{j_{1}} f^{j_{2}, j} I_{\left(j_{1}, j_{2}\right)} \\
& +\sum_{j_{1}, j_{2}, j_{3}=0}^{m} L^{j_{1}} L^{j_{2}} f^{j_{3}, k}\left(t_{0}, x\left(t_{0}\right)\right) I_{\left(j_{1}, j_{2}, j_{3}\right)}+O\left(\Delta^{4}\right)
\end{aligned}
$$

for $k=1, \ldots, d$, where we have written $I_{(j)}$ for $I_{(j), t_{0}, t_{0}+\Delta}$ and so on.

\section{Taylor schemes}

Using the Taylor approximation from the previous section we now construct numerical schemes by iterating Taylor approximations, or suitable derivative free approximations of those, over a partition of the time interval under interest. Schemes of arbitrary higher order $N=1,2, \ldots$ can be constructed by truncating the Taylor approximation corresponding to the the hierarchical set $\Gamma_{N}$. Here we assume that the multiple control integrals $I_{\alpha}$ are at our disposal; in Section 9 we shall describe how these integrals can be approximated.

Let $\left\{t_{0}, t_{1}, \ldots, t_{n}, \ldots,\right\}$ be a partition of the time interval $\left[t_{0}, T\right]$ with stepsizes $\Delta_{n}$ $=t_{n+1}-t_{n}$ and maximal step size $\Delta:=\max _{n} \Delta_{n}$. In the general multi-dimensional case $d, m=1,2, \ldots$ for $N=1,2,3, \ldots$ we define the Taylor scheme of order $N$ for the affinely controlled differential equation (6) is given componentwise by

$$
X_{n+1}^{k}=X_{n}^{k}+\sum_{\alpha \in \Gamma_{N} \backslash\{\odot\}} F_{\alpha}^{k}\left(t_{n}, X_{n}\right) I_{\alpha, t_{n}, t_{n+1}}
$$

with the coefficient functions $F_{\alpha}^{k}$ corresponding to $F(t, x) \equiv x^{k}$ for $k=1, \ldots, d$ and the multiple control integrals

$$
I_{\alpha, t_{n}, t_{n+1}}=\int_{t_{n}}^{t_{n+1}} \int_{t_{n}}^{s_{l}} \cdots \int_{t_{n}}^{s_{2}} u^{j_{1}}\left(s_{1}\right) \cdots u^{j_{l}}\left(s_{l}\right) d s_{1} \cdots d s_{l} .
$$

By standard arguments [12] it follows from (20) that the global discretization error is of order $N$ when the drift and control coefficients $f^{0}, f^{1}, \ldots, f^{m}$ of the differential equation (6) are $N$ times continuously differentiable.

In writing out the Taylor schemes below, we shall distinguish the purely uncontrolled integrals, that is with multi-indices $(0),(0,0),(0,0,0), \ldots$ from the others, since no special effort is required for their evaluation. 


\subsection{The Euler scheme}

The Euler approximation is the simplest nontrivial Taylor scheme. It corresponds to the hierarchical set $\Gamma_{1}$ and has the convergence order $N=1$. It is given componentwise by

$$
X_{n+1}^{k}=X_{n}^{k}+f^{0, k}\left(t_{n}, X_{n}\right) \Delta_{n}+\sum_{j=1}^{m} f^{j, k}\left(t_{n}, X_{n}\right) I_{(j), t_{n}, t_{n+1}}
$$

for $k=1, \ldots, d$, where

$$
\Delta_{n}=t_{n+1}-t_{n}=\int_{t_{n}}^{t_{n+1}} d s \quad \text { and } \quad I_{(j), t_{n}, t_{n+1}}=\int_{t_{n}}^{t_{n+1}} u^{j}(s) d s, \quad j=1, \ldots, m .
$$

\subsection{The Taylor scheme of order 2}

The $k$ th component of the Taylor scheme of order 2 is given by

$$
\begin{aligned}
X_{n+1}^{k}=X_{n}^{k} & +f^{0, k}\left(t_{n}, X_{n}\right) \Delta_{n}+\sum_{j=1}^{m} f^{j, k}\left(t_{n}, X_{n}\right) I_{(j), t_{n}, t_{n+1}} \\
& +\frac{1}{2} L^{0} f^{0, k}\left(t_{n}, X_{n}\right) \Delta_{n}^{2}+\sum_{\substack{j_{1}, j_{2}=0 \\
j_{1}+j_{2} \neq 0}}^{m} L^{j_{1}} f^{j_{2}, k}\left(t_{n}, X_{n}\right) I_{\left(j_{1}, j_{2}\right), t_{n}, t_{n+1}}
\end{aligned}
$$

for $k=1, \ldots, d$.

\subsection{The Taylor scheme of order 3}

The Taylor scheme of order 3 is given componentwise by

$$
\begin{aligned}
X_{n+1}^{k}=X_{n}^{k} & +f^{0, k}\left(t_{n}, X_{n}\right) \Delta_{n}+\sum_{j=1}^{m} f^{j, k}\left(t_{n}, X_{n}\right) I_{(j), t_{n}, t_{n+1}} \\
& +\frac{1}{2} L^{0} f^{0, k}\left(t_{n}, X_{n}\right) \Delta_{n}^{2}+\sum_{\substack{j_{1}, j_{2}=0 \\
j_{1}+j_{2} \neq 0}}^{m} L^{j_{1}} f^{j_{2}, k}\left(t_{n}, X_{n}\right) I_{\left(j_{1}, j_{2}\right), t_{n}, t_{n+1}} \\
& +\frac{1}{6} L^{0} L^{0} f^{0, k}\left(t_{n}, X_{n}\right) \Delta_{n}^{3}+\sum_{\substack{j_{1}, j_{2}, j_{3}=0 \\
j_{1}+j_{2}+j_{3} \neq 0}}^{m} L^{j_{2}} f^{j_{3}, k}\left(t_{n}, X_{n}\right) I_{\left(j_{1}, j_{2}, j_{3}\right), t_{n}, t_{n+1}}
\end{aligned}
$$

for $k=1, \ldots, d$. 


\section{Derivative-free schemes}

A disadvantage of Taylor schemes is that the derivatives of various orders of the drift and control coefficients must be first derived and then evaluated at each step. In the past this made the implementation of such schemes a complicated undertaking, but this is no longer such a difficulty these days with symbolic manipulators [3]. Nevertheless it is useful to have approximations and schemes that avoid the use of derivatives of the drift and control coefficients in much the same way that Runge-Kutta schemes do in the more traditional setting since these often have other computational advantages.

In this section we shall illustrate how such derivative-free schemes can be derived. These could also be called Runge-Kutta schemes, but it must be emphasized that they are not simply heuristic adaptations of the traditional Runge-Kutta schemes to affinely controlled differential systems, which will usually not attain their traditionally asserted order in this context.

Since the Euler or Taylor scheme of order 1 contains no derivatives of $f^{0} f^{1}, \ldots$, $f^{m}$, we consider the second order Taylor scheme (27) in the scalar autonomous case with a single control, that is with $d=m=1$. Here the affinely controlled differential equation is given by (2) and the Taylor scheme by

$$
\begin{aligned}
X_{n+1}=X_{n}+ & f^{0}\left(X_{n}\right) \Delta_{n}+f^{1}\left(X_{n}\right) I_{(1), t_{n}, t_{n+1}} \\
& +\frac{1}{2} L^{0} f^{0}\left(X_{n}\right) \Delta_{n}^{2}+L^{0} f^{1}\left(X_{n}\right) I_{(0,1), t_{n}, t_{n+1}} \\
& +L^{1} f^{0}\left(X_{n}\right) I_{(1,0), t_{n}, t_{n+1}}+L^{1} f^{1}\left(X_{n}\right) I_{(1,1), t_{n}, t_{n+1}},
\end{aligned}
$$

or, using a dash ' to denote differentiation with respect to $x$, by

$$
\begin{aligned}
X_{n+1}=X_{n}+ & f^{0}\left(X_{n}\right) \Delta_{n}+f^{1}\left(X_{n}\right) I_{(1), t_{n}, t_{n+1}} \\
& +\frac{1}{2} f^{0}\left(X_{n}\right) f^{0 \prime}\left(X_{n}\right) \Delta_{n}^{2}+f^{0}\left(X_{n}\right) f^{1 \prime}\left(X_{n}\right) I_{(0,1), t_{n}, t_{n+1}} \\
& +f^{1}\left(X_{n}\right) f^{0 \prime}\left(X_{n}\right) I_{(1,0), t_{n}, t_{n+1}}+f^{1}\left(X_{n}\right) f^{1 \prime}\left(X_{n}\right) I_{(1,1), t_{n}, t_{n+1}},
\end{aligned}
$$

By the ordinary Taylor expansion we have

$$
f^{j}(x) f^{i \prime}(x)=\frac{1}{\Delta}\left(f^{i}\left(x+f^{j}(x) \Delta\right)-f^{i}(x)\right)+O(\Delta),
$$

so the $(i, j)$ term in the above Taylor scheme reads

$$
L^{i} f^{j}\left(X_{n}\right) I_{(1,0), t_{n}, t_{n+1}}=f^{j}\left(X_{n}\right) f^{i \prime}\left(X_{n}\right) I_{(i, j), t_{n}, t_{n+1}}
$$




$$
\begin{aligned}
& =\left(\frac{1}{\Delta_{n}}\left(f^{i}\left(X_{n}+f^{j}\left(X_{n}\right) \Delta_{n}\right)-f^{i}\left(X_{n}\right)\right)+O\left(\Delta_{n}\right)\right) I_{(i, j), t_{n}, t_{n+1}} \\
& =\frac{1}{\Delta_{n}}\left(f^{i}\left(X_{n}+f^{j}\left(X_{n}\right) \Delta_{n}\right)-f^{i}\left(X_{n}\right)\right) I_{(i, j), t_{n}, t_{n+1}}+O\left(\Delta_{n}^{3}\right)
\end{aligned}
$$

since $O\left(\Delta_{n}\right) I_{(i, j), t_{n}, t_{n+1}}=O\left(\Delta_{n}^{3}\right)$. The remainder here is of the same order as the local discretization error, so we can replace the term on the left by that on the right without reducing the global order of the resulting scheme. In this way we obtain the second order derivative-free scheme

$$
\begin{aligned}
X_{n+1}=X_{n}+ & f^{0}\left(X_{n}\right) \Delta_{n}+f^{1}\left(X_{n}\right) I_{(1), t_{n}, t_{n+1}} \\
& +\frac{1}{2}\left(f^{0}\left(X_{n}+f^{0}\left(X_{n}\right) \Delta_{n}\right)-f^{0}\left(X_{n}\right)\right) \Delta_{n} \\
& +\frac{1}{\Delta_{n}} \sum_{\substack{i, j=0 \\
i+j \neq 0}}^{1}\left(f^{i}\left(X_{n}+f^{j}\left(X_{n}\right) \Delta_{n}\right)-f^{i}\left(X_{n}\right)\right) I_{(i, j), t_{n}, t_{n+1}} \\
=X_{n}+ & \frac{1}{2} f^{0}\left(X_{n}\right) \Delta_{n}+f^{1}\left(X_{n}\right) I_{(1), t_{n}, t_{n+1}} \\
& +\frac{1}{2} f^{0}\left(X_{n}+f^{0}\left(X_{n}\right) \Delta_{n}\right) \Delta_{n} \\
& +\frac{1}{\Delta_{n}} \sum_{\substack{i, j=0 \\
i+j \neq 0}}^{1}\left(f^{i}\left(X_{n}+f^{j}\left(X_{n}\right) \Delta_{n}\right)-f^{i}\left(X_{n}\right)\right) I_{(i, j), t_{n}, t_{n+1}}
\end{aligned}
$$

in the scalar autonomous case with a single control, i.e. $d=m=1$. This was also obtained by Ferretti [5] when the control coefficient $f^{1}$ was equal to a constant.

The vector version of the second order derivative-free scheme for an autonomous affine control system has $k$ th component given by

$$
\begin{aligned}
X_{n+1}^{k}=X_{n}^{k}+ & \frac{1}{2} f^{0, k}\left(X_{n}\right) \Delta_{n}+\sum_{j=1}^{m} f^{j, k}\left(X_{n}\right) I_{(j), t_{n}, t_{n+1}} \\
& +\frac{1}{2} f^{0, k}\left(X_{n}+f^{0}\left(X_{n}\right) \Delta_{n}\right) \Delta_{n} \\
& +\frac{1}{\Delta_{n}} \sum_{\substack{i, j=0 \\
i+j \neq 0}}^{m}\left(f^{i, k}\left(X_{n}+f^{j}\left(X_{n}\right) \Delta_{n}\right)-f^{i, k}\left(X_{n}\right)\right) I_{(i, j), t_{n}, t_{n+1}}
\end{aligned}
$$

for $k=1, \ldots, d$. In the usual ODE case, that is with $f^{j}(x) \equiv 0$ for $j=1, \ldots, m$, this is just the second order Runge-Kutta scheme known as the Heun scheme. 
This principle can be extended to obtain higher order derivative-free schemes. See [13] for analogous higher order derivative-free schemes for the stochastic case.

\section{Simplifications with additive or commutative con- trol}

The Taylor schemes (24) simplify considerable when the drift and control coefficients $f^{0}$, $f^{1}, \ldots, f^{m}$ of the affinely controlled differential equation (6) satisfy special properties. For example, if the control coefficients $f^{1}, \ldots, f^{m}$ are all constants or depend just on $t$, we shall say that the control system has additive control. In this case all of the spatial derivatives of these control coefficients vanish and, hence, so do the corresponding higher order terms. For example, the second order Taylor scheme (27) then reduces to

$$
\begin{gathered}
X_{n+1}^{k}=X_{n}^{k}+f^{0, k}\left(t_{n}, X_{n}\right) \Delta_{n}+\sum_{j=1}^{m} f^{j, k}\left(t_{n}, X_{n}\right) I_{(j), t_{n}, t_{n+1}} \\
+\sum_{j=0}^{m} L^{j} f^{0, k}\left(t_{n}, X_{n}\right) I_{(j, 0), t_{n}, t_{n+1}}
\end{gathered}
$$

for $k=1, \ldots, d$.

Another major simplification occurs under commutative control, that is when the drift and control coefficients satisfy

$$
L^{i} f^{j, k}(t, x) \equiv L^{j} f^{i, k}(t, x) \quad \text { for all } i, j=0,1, \ldots, m .
$$

Then, by the generalized integration-by-parts identities

$$
I_{(i, j), t_{n}, t_{n+1}}+I_{(j, i), t_{n}, t_{n+1}}=I_{(i), t_{n}, t_{n+1}} I_{(j), t_{n}, t_{n+1}}, \quad i, j=0,1, \ldots, m
$$

the sum of terms

$$
L^{i} f^{j, k}\left(t_{n}, X_{n}\right) I_{(i, j), t_{n}, t_{n+1}}+L^{j} f^{i, k}\left(t_{n}, X_{n}\right) I_{(j, i), t_{n}, t_{n+1}}
$$

simplifies to

$$
L^{i} f^{j, k}\left(t_{n}, X_{n}\right) I_{(i), t_{n}, t_{n+1}} I_{(j), t_{n}, t_{n+1}},
$$

which involves more easily computed multiple control integrals of lower multiplicity. Note that this condition is similar to the one considered in [14], where the effect of time discretization of the control function is investigated and a second order scheme for the approximation of the reachable set is obtained. 


\section{Approximation of multiple control integrals}

In control theory the computation of a trajectory corresponding to a single control function as well as the computation of the reachable set corresponding to the trajectories of all possible control functions are both of considerable interest, see [2]. Both require the evaluation or approximation multiple control integrals appearing in the numerical schemes that a have been proposed above. Here we suggest several ways this can be done.

\subsection{Averaging multiple integrals of a single control function}

A multiple control integral $I_{\alpha, t_{n}, t_{n+1}}=I_{\alpha, u, t_{n}, t_{n+1}}$ for a measurable control function $u$ taking values in $\mathbf{U}_{m}$ can often be evaluated explicitly using, for example, a symbolic manipulator such as MAPLE. For complicated multiple integrals, however, this might become very slow, so it could be more convenient to use a numerical approximation instead. In this section we show how this can be done by an averaging strategy, an approach adopted from [8], but with the major difference that here we are dealing with measurable instead of the Hölder continuous functions considered in [8]. This difference will make it necessary to assume certain knowledge about the integrals of the control function $u$ over short time intervals.

The following Lemma provides the main estimate for our purpose. As above we use the convention that $u^{0}(t) \equiv 1$.

Lemma 2 Consider a measurable control function $u:[0, \Delta] \rightarrow \mathbf{U}_{m}$, some $P \in \mathbb{N}$, $\beta=\Delta / P>0$, and define

$$
\hat{u}_{k}^{j}:=\int_{(k-1) \beta}^{k \beta} u^{j}(t) d t
$$

for $i=0, \ldots, m, k=1, \ldots, P$. Then

$$
I_{\alpha, u, 0, \Delta}=\sum_{k_{1}=1}^{P} \sum_{k_{2}=1}^{k_{1}} \cdots \sum_{k_{l}=1}^{k_{l-1}} \hat{u}_{k_{1}}^{j_{1}} \cdots \hat{u}_{k_{l}}^{j_{l}}+O\left(\beta \Delta^{l-1}\right)
$$

for all $l \geq 2$ and all $\alpha=\left(j_{1}, \ldots, j_{l}\right)$.

Proof: We will show by induction over $l$ that

$$
I_{\left(j_{1}, \ldots, j_{l}\right), u, 0, \epsilon}=\sum_{k_{1}=1}^{[P \epsilon / \Delta]} \sum_{k_{2}=1}^{k_{1}} \cdots \sum_{k_{l}=1}^{k_{l-1}} \hat{u}_{k_{1}}^{j_{1}} \cdots \hat{u}_{k_{l}}^{j_{l}}+O\left(\beta \Delta^{l-1}\right)
$$

for an arbitrary $\epsilon \in(0, \Delta]$, where $[r]$ denotes the smallest integer greater or equal to $r \in \mathbb{R}$. This will imply the assertion on setting $\epsilon=\Delta$. 
For $l=1$ the assertion follows immediately from the definition of the $\hat{u}_{k}^{j}$. Now consider $\left(j_{0}, j_{1}, \ldots, j_{l}\right)$ with $l \geq 2$. Then

$$
I_{\left(j_{0}, j_{1}, \ldots, j_{l}\right), u, 0, \epsilon}=\int_{0}^{\epsilon} u^{j_{0}}(t) I_{\left(j_{1}, \ldots, j_{l}\right), u, 0, t} d t
$$

and by the induction assumption we can proceed to obtain

$$
\begin{aligned}
& =\int_{0}^{\epsilon} u^{j_{0}}(t)\left(\sum_{k_{1}=1}^{[P t / \Delta]} \sum_{k_{2}=1}^{k_{1}} \cdots \sum_{k_{l}=1}^{k_{l-1}} \hat{u}_{k_{1}}^{j_{1}} \cdots \hat{u}_{k_{l}}^{j_{l}}+O\left(\beta \Delta^{l-1}\right)\right) d t \\
& =\int_{0}^{\epsilon} u^{j_{0}}(t)\left(\sum_{k_{1}=1}^{[P t / \Delta]} \sum_{k_{2}=1}^{k_{1}} \cdots \sum_{k_{l}=1}^{k_{l-1}} \hat{u}_{k_{1}}^{j_{1}} \cdots \hat{u}_{k_{l}}^{j_{l}}\right) d t+O\left(\beta \Delta^{l}\right) \\
& =\left(\sum_{k_{0}=1}^{[P \epsilon / \Delta]} \hat{u}_{k_{0}}^{j_{0}}+O(\beta)\right) \underbrace{\left(\sum_{k_{1}=1}^{k_{0}} \sum_{k_{2}=1}^{k_{1}} \cdots \sum_{k_{l}=1}^{k_{l-1}} \hat{u}_{k_{1}}^{j_{1}} \cdots \hat{u}_{k_{l}}^{j_{l}}\right)}_{=O\left(\Delta^{l}\right)}+O\left(\beta \Delta^{l}\right) \\
& =\sum_{k_{0}=1}^{[P \epsilon / \Delta]} \sum_{k_{1}=1}^{k_{0}} \cdots \sum_{k_{l}=1}^{k_{l-1}} \hat{u}_{k_{0}}^{j_{0}} \cdots \hat{u}_{k_{l}}^{j_{l}}+O\left(\beta \Delta^{l}\right)
\end{aligned}
$$

which finishes the proof.

Assuming that the values $\hat{u}_{k}^{j}$ are known, based on this estimate one can use the following strategy for approximating $I_{\alpha, u, t, t+\Delta}$ : Given some step size $\Delta>0$, a scheme of order $N \in \mathbb{N}$ and some multi-index $\alpha$ with $l(\alpha) \geq 2$, fix $\beta>0$ such that $\beta \leq \Delta^{N+2-l(\alpha)}$, and approximate the corresponding control integrals by $(34)$; for $l(\alpha)=1$, knowledge of $\hat{u}_{k}^{j}$ allows an exact evaluation. Then the Lemma 2 ensures that $I_{\alpha, t, t+\Delta}$ is approximated with an error of order $\Delta^{N+1}$ thus maintaining the local, and hence global, order of the scheme.

Note that on any fixed time interval the number of computations involving the $\hat{u}_{k}^{j}$ is of the order of $1 / \Delta^{N}$, and hence grows with the order of the scheme as $\Delta \rightarrow 0$. On the other hand, the number of evaluations of the $f_{i}$ (which in general will be the more expensive part, especially when the dimension $d$ of the state space is high) only grows like $1 / \Delta$, hence linearly. This difference in the computational cost is typical for averaged schemes, see also [8].

\subsection{Approximating the set of all possible multiple control in- tegrals}

In many applications one is interested in simulating the whole set of possible trajectories, for example, as in solving numerically a Hamilton-Jacobi equation related to optimal control (e.g. $[4,7])$ or in the computation of a reachable set (e.g. $[2,10,11])$. 
This requires knowledge of the set of multiple control integrals for all possible control values in the scheme that will be used.

For $\mathcal{A}=\left\{\alpha_{1}, \ldots, \alpha_{p}\right\} \subset \mathcal{M}_{m}$ the explicit determination of the set

$$
\left.\mathcal{I}_{\mathcal{A}, \mathbf{U}_{m}, t, t+\Delta}:=\bigcup\left\{\left(I_{\alpha_{1}, u, t, t+\Delta}, \ldots, I_{\alpha_{p}, u, t, t+\Delta}\right) \mid \text { all measurable } u: \rightarrow \mathbf{U}_{m}\right)\right\} \subset \mathbb{R}^{r m}
$$

of all possible values of the multiple control integrals is rather complicated and beyond the scope of this paper. For results in this direction we refer to [5], where only the (easier but still quite complicated) cases $\alpha=(0, \ldots, 0, i)$ and $\alpha=(i, 0, \ldots, 0), i=$ $1, \ldots, m$, which are all that is needed in the restricted class of additively controlled systems considered there (cf. Section 8), are treated. Note, however, that by (33) these results also suffice for the computation of $\mathcal{I}_{\mathcal{A}, \mathbf{U}_{m}, t, t+\Delta}$ for second order approximations for systems with one-dimensional control (the so called single input systems), as well as for commutative control systems and the special case where only the control coefficients $f_{1}, \ldots, f_{m}$ commute, see again Section 8 .

In the general case we propose the following simple numerical procedure for an approximation $\tilde{\mathcal{I}} \subset \mathcal{I}_{\mathcal{A}, t, t+\Delta, \mathbf{U}_{m}}$ satisfying

$$
\inf _{I \in \tilde{\mathcal{I}}} \sup _{I^{\prime} \in \mathcal{I}_{\mathcal{A}, \mathbf{U}_{m}}, t, t+\Delta}\left\|I-I^{\prime}\right\| \leq K \Delta^{N+1}
$$

for all $\Delta \in\left(0, \Delta_{0}\right]$ and some $K=K\left(\Delta_{0}\right)>0$ independent of $\Delta$ and thus maintaining the order of the scheme.

Step 1: Choose some time step $\Delta$ and some desired order $N \in \mathbb{I}$. Pick a scheme of order $N$ and the corresponding set of multi-indices $\mathcal{A}=\Gamma_{N}$.

Step 2: Consider a discrete set $\tilde{\mathbf{U}}_{m} \subset \mathbf{U}_{m}$ satisfying

$$
\inf _{\tilde{u} \in \tilde{\mathbf{U}}_{m}} \sup _{u \in \mathbf{U}_{m}}\|\tilde{u}-u\| \leq \Delta^{N}
$$

$P \in \mathbb{N}$ with $\beta:=\Delta / P \leq \Delta^{N+1}$ and the space $\tilde{\mathcal{U}}$ of measurable control functions satisfying

$$
\frac{1}{\beta} \int_{(k-1) \beta}^{k \beta} u(t) d t \in \tilde{\mathbf{U}}_{m}
$$

for all $k \in\{1,2, \ldots, P\}$, which can be identified with $\tilde{\mathbf{U}}_{m}^{P}$

Step 3: Compute the approximations (34) to the multiple control integrals for all $v \in \tilde{\mathcal{U}}$.

This way for each admissible control function $u(\cdot)$ we find a control function $v(\cdot) \in \tilde{\mathcal{U}}$ such that

$$
\left|\hat{u}_{k}^{j}-\hat{v}_{k}^{j}\right|=\left|\int_{(k-1) \beta}^{k \beta} u^{j}(t) d t-\int_{(k-1) \beta}^{k \beta} v^{j}(t) d t\right| \leq \beta \Delta^{N}
$$


implying for $l \geq 1$

$$
\left|\sum_{k_{1}=1}^{P} \sum_{k_{2}=1}^{k_{1}} \cdots \sum_{k_{l}=1}^{k_{l-1}} \hat{u}_{k_{1}}^{j_{1}} \cdots \hat{u}_{k_{l}}^{j_{l}}-\sum_{k_{1}=1}^{P} \sum_{k_{2}=1}^{k_{1}} \cdots \sum_{k_{l}=1}^{k_{l-1}} \hat{v}_{k_{1}}^{j_{1}} \cdots \hat{v}_{k_{l}}^{j_{l}}\right| \leq \frac{P^{l}}{l !} C \beta^{l} \Delta^{N} \leq C \Delta^{N+l}
$$

for some constant $C>0$ independent of $\Delta$ and $\beta$. For $\Delta \in\left(0, \Delta_{0}\right]$ we have $\Delta^{N+l} \leq$ $\Delta_{0}^{l-1} \Delta^{N+1}$ and thus using Lemma 2 it is straightforward to verify that this set indeed has the desired approximation property.

Note that in many algorithms the numerical scheme has to be evaluated many times at different state space points $x$, but since $\mathcal{I}_{\mathcal{A}, \mathbf{U}_{m}, t, t+\Delta}$ does not depend on $x$ the computation of $\tilde{\mathcal{I}}$ needs to be done just once at the beginning of the algorithm.

There might be a number of more efficient ways for the construction of an approximating set $\tilde{\mathcal{I}}$. In particular, in optimization problems it might be sufficient to approximate the extremal points of the (convex) set $\mathcal{I}_{\mathcal{A}, \mathbf{U}_{m}, t, t+\Delta}$ and use some optimization strategy on this set instead of using all the points "inside" $\tilde{\mathcal{I}}$. For bang-bang optimal control it suffices to construct $\tilde{\mathcal{I}}$ by choosing $\tilde{\mathbf{U}}_{m}$ as the set of extremal points of the convex set $\mathbf{U}_{m}$. Such strategies, however, depend strongly on the structure of the problem for which the numerical approximation of the controlled trajectories is needed.

\section{A numerical example}

We have tested the Euler and Heun Schemes from Sections 6 and 7 with the 2 dimensional system with a single control

$$
\frac{d x(t)}{d t}=f^{0}(x(t))+u(t) f^{1}(x(t)):=\left(\begin{array}{c}
x_{2}(t) \\
0
\end{array}\right)+u(t)\left(\begin{array}{c}
-x_{2}(t) \\
1
\end{array}\right)
$$

with control function $u(t)=\sin (100 / t)$ and initial value $x_{0}=(0,0)^{T}$. The resulting schemes have been simplified using the identity (33) such that the only remaining control integrals were $I_{(1), 0, t}$ and $I_{(0,1), 0, t}$, which have been evaluated using MAPLE. Note that the exact solution for this equation is easily verified to be

$$
x(t)=I_{(1,0), 0, t}-I_{(1,1), 0, t}, \quad y(t)=I_{(1), 0, t} .
$$

The equation was solved on the interval $[0,1]$ with timestep $\Delta=1 / N$ and $N=50$, $100, \ldots, 400$. Figure 1 shows the resulting errors $\sup _{n=1, \ldots, N}\left\|x_{n}-x(n \Delta)\right\|$ for the Heun and the Euler scheme. The left figure shows the error over $N$ in a linear scale, the right figure shows the error over $\Delta$ in a log-log scale. Note that the two small values (clearly visible in the log-log plot) are due to cancellation of local errors and hence are better than expected. 

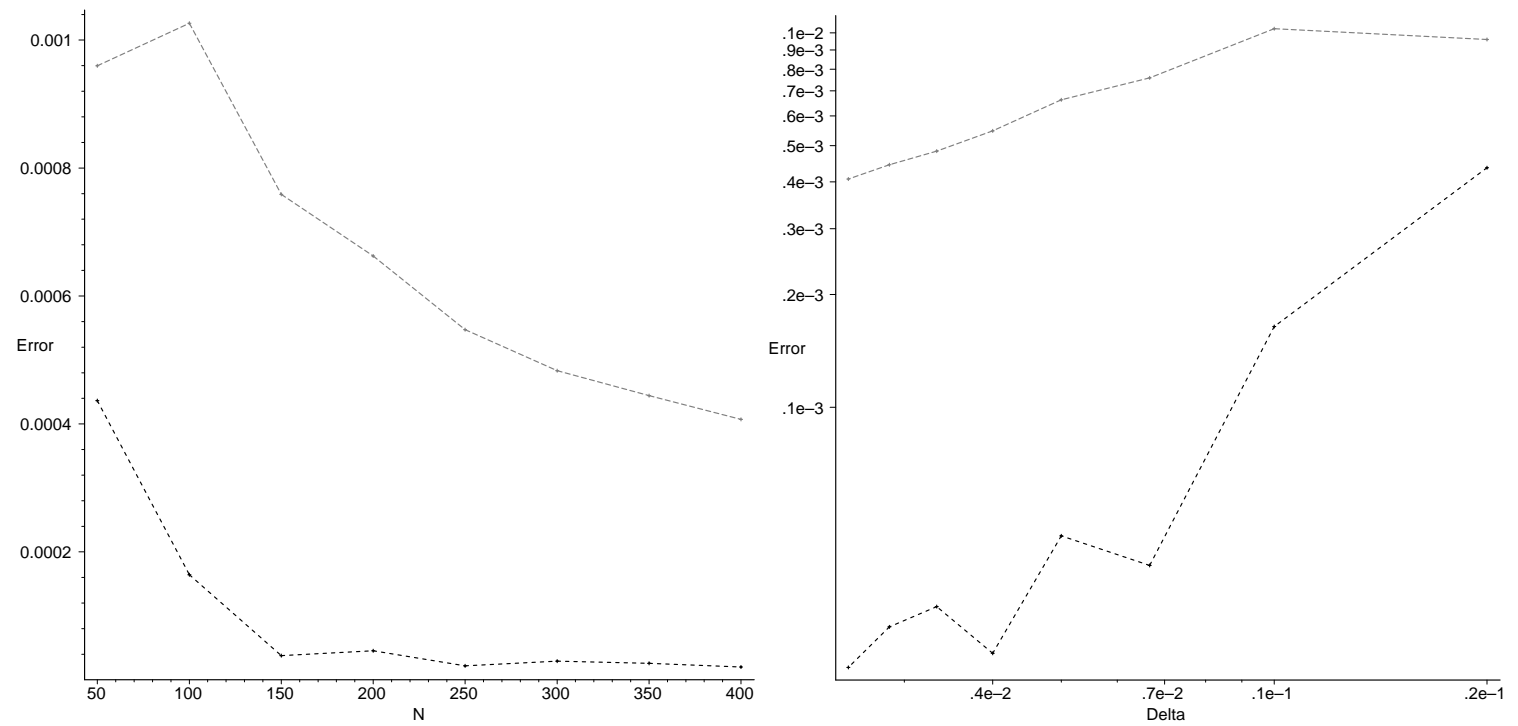

Figure 1: Global error for Heun (black) and Euler (grey) schemes, linear and log-log

The Figures 2 and 3 show the $x_{1}$ component of the exact solution, of the Heun and of the Euler scheme for $N=100$ and $N=400$, respectively.

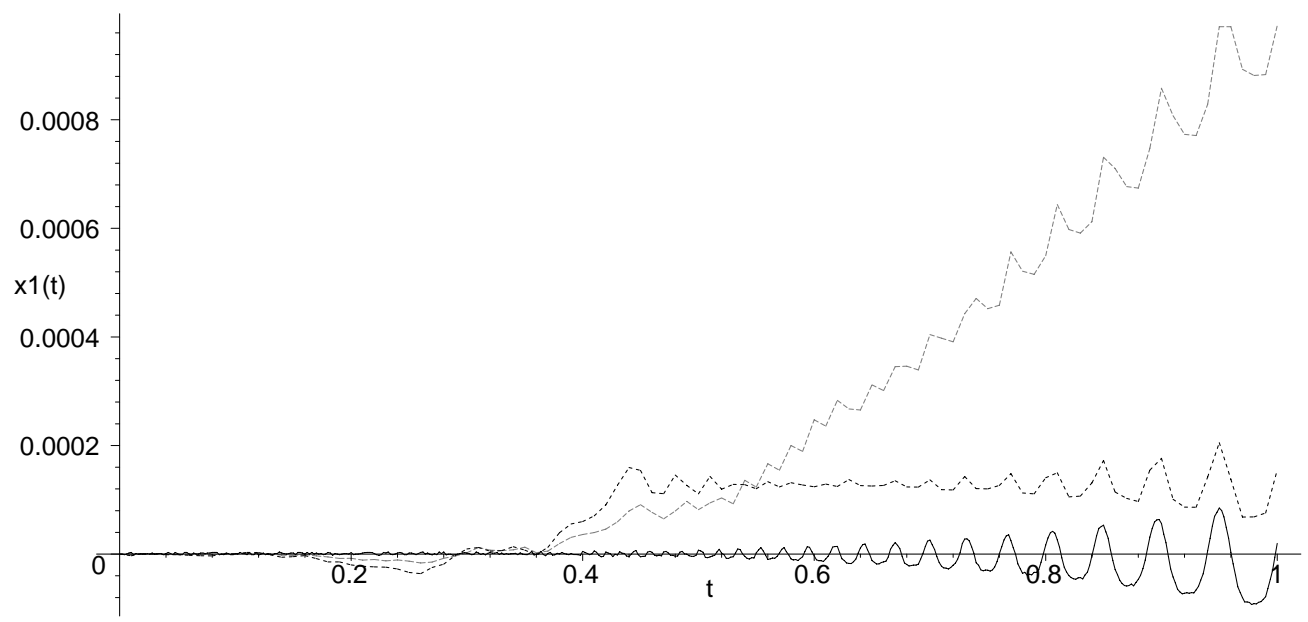

Figure 2: Exact (solid), Heun (black dashed) and Euler (grey dashed) solution for $N=100$ 


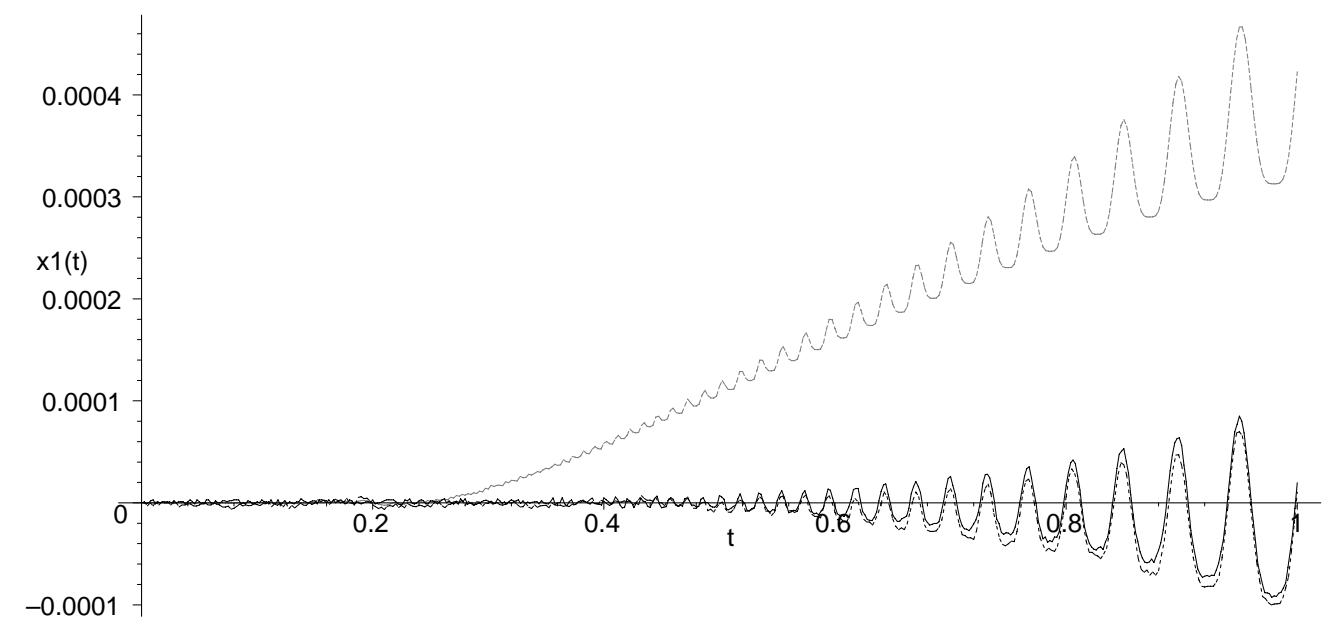

Figure 3: Exact (solid), Heun (black dashed) and Euler (grey dashed) solution for $N=400$

\section{References}

[1] L. Arnold, Random Dynamical Systems. Springer-Verlag, Heidelberg, 1998.

[2] F. Colonius and W. Kliemann, The Dynamics of Control, Birkhäuser, Boston, 2000 .

[3] S. Cyganowski, P. E. Kloeden and T. Pohl, MAPLE for stochastic differential equations WIAS Berlin, Preprint Nr. 453, 1998. Availability: Postscript 467 KB, http : //www.wias - berlin.de

[4] M. Falcone and R. Ferretti, Discrete time high-order schemes for viscosity solutions of Hamilton-Jacobi-Bellman equations. Numer. Math. 67 (1994), 315-344.

[5] R. Ferretti, Higher-order approximations of linear control systems via RungeKutta schemes. Computing 58 (1997), 351-364.

[6] R.A. Gordon, The Integrals of Lebesgue, Denjoy, Perron, and Henstock. Graduate Studies in Mathematics, Vol. 4, American Mathematical Society, Providence, RI, 1994.

[7] L. Grüne, An adaptive grid scheme for the discrete Hamilton-Jacobi-Bellman equation. Numer. Math. 75 (1997), 319-337.

[8] L. Grüne and P.E. Kloeden, Pathwise approximation of random ordinary differential equations. Preprint 26/99, DFG-Schwerpunkt "Ergodentheorie, Analysis und effiziente Simulation dynamischer Systeme". Submitted. 
[9] A. Isidori, Nonlinear Control Systems. An Introduction. Second edition, SpringerVerlag, Heidelberg, 1995.

[10] G. Häckl, Numerical approximation of reachable sets and control sets. Random Comput. Dyn. 1 (1992-1993), 371-394.

[11] G. Häckl, Reachable Sets, Control Sets and Their Computation. Augsburger Mathematisch-Naturwissenschaftliche Schriften, Vol. 7, Wißner-Verlag, Augsburg, 1995.

[12] E. Hairer, S.P. Norsett and G. Wanner, Solving Ordinary Differential Equations I. Springer-Verlag, Heidelberg, 1988.

[13] P.E. Kloeden and E. Platen, Numerical Solution of Stochastic Differential Equations. Springer-Verlag, Heidelberg, 1992 (3rd revised and updated printing, 1999).

[14] V. Veliov, On the time discretization of control systems, SIAM J. Control Optim. 35 (1997), 1470-1486. 